\title{
Atypical Inflammatory Syndrome Triggered by SARS-CoV-2 in Infants with Down Syndrome
}

\author{
Louise Malle ${ }^{1,2,3,4,5} \cdot$ Paul Bastard 6 ,7,8 . Andrea Martin-Nalda ${ }^{9,10} \cdot$ Taya Carpenter $^{11}$. Douglas Bush ${ }^{2}$. \\ Roosheel Patel $1^{1,2,3,4,5} \cdot$ Roger Colobran ${ }^{12,13} \cdot$ Pere Soler-Palacin ${ }^{9,10}$. Jean-Laurent Casanova ${ }^{6,7,8,14}$. \\ Melissa Gans ${ }^{11,15,16}$. Jacques G. Rivière ${ }^{9,10}$ • Dusan Bogunovic ${ }^{1,2,3,4,5}$ (1)
}

Received: 19 March 2021 / Accepted: 25 May 2021 / Published online: 5 June 2021

(c) The Author(s), under exclusive licence to Springer Science+Business Media, LLC, part of Springer Nature 2021

\begin{abstract}
While adults with Down syndrome (DS) are at increased risk of severe COVID-19 pneumonia, little is known about COVID19 in children with DS. In children without DS, SARS-CoV-2 can rarely cause severe COVID-19 pneumonia, or an even rarer and more typically pediatric condition, multisystem inflammatory syndrome in children (MIS-C). Although the underlying mechanisms are still unknown, MIS-C is thought to be primarily immune-mediated. Here, we describe an atypical, severe form of MIS-C in two infant girls with DS who were hospitalized for over 4 months. Immunological evaluation revealed pronounced neutrophilia, B cell depletion, increased circulating IL-6 and IL-8, and elevated markers of immune activation ICAM1 and FcyRI. Importantly, uninfected children with DS presented with similar but less stark immune features at steady state, possibly explaining risk of further uncontrolled inflammation following SARS-CoV-2 infection. Overall, a severe, atypical form of MIS-C may occur in children with DS.
\end{abstract}

Keywords Down syndrome $\cdot$ COVID-19 $\cdot$ MIS-C $\cdot$ inborn errors of immunity

Dusan Bogunovic

Dusan.Bogunovic@mssm.edu

1 Center for Inborn Errors of Immunity, Icahn School of Medicine At Mount Sinai, New York, NY, USA

2 Department of Pediatrics, Icahn School of Medicine At Mount Sinai, New York, NY, USA

3 Mindich Child Health and Development Institute, Icahn School of Medicine At Mount Sinai, New York, NY, USA

4 Precision Immunology Institute, Icahn School of Medicine At Mount Sinai, New York, NY, USA

5 Department of Microbiology, Icahn School of Medicine At Mount Sinai, New York, NY, USA

6 Laboratory of Human Genetics of Infectious Diseases, Necker Branch, INSERM U1163, Necker Hospital for Sick Children, Paris, France

7 University of Paris, Imagine Institute, Paris, France

8 St. Giles Laboratory of Human Genetics of Infectious Diseases, Rockefeller Branch, The Rockefeller University, New York, NY, USA

9 Pediatric Infectious Diseases and Immunodeficiencies Unit, Hospital Universitari Vall D'Hebron (HUVH), Vall d'Hebron Barcelona Hospital Campus, Barcelona, Catalonia, Spain
10 Infection in Immunocompromised Pediatric Patients Research Group, Vall D'Hebron Institut de Recerca (VHIR), Hospital Universitari Vall D'Hebron (HUVH), Vall d'Hebron Barcelona Hospital Campus, Barcelona, Catalonia, Spain

11 Department of Pediatrics, Maria Fareri Children's Hospital At Westchester Medical Center, Valhalla, NY, USA

12 Immunology Division, Hospital Universitari Vall D’Hebron (HUVH), Diagnostic Immunology Research Group, Vall D'Hebron Institut de Recerca (VHIR), Vall D'Hebron Barcelona Hospital Campus, Department of Cell Biology, Physiology and Immunology, Universitat Autònoma de Barcelona (UAB), Barcelona, Catalonia, Spain

13 Genetics Department, Hospital Universitari Vall D’Hebron (HUVH), Vall D’Hebron Barcelona Hospital Campus, Barcelona, Catalonia, Spain

14 Howard Hughes Medical Institute, New York, NY, USA

15 Division of Pediatric Pulmonology, Allergy and Immunology and Sleep Medicine, Boston Children's Health Physicians, Valhalla, NY, USA

16 New York Medical College, Valhalla, NY, USA 


\section{Introduction}

While adults have suffered the highest rates of morbidity and mortality from COVID-19 pneumonia, children are relatively spared from this manifestation of SARS-CoV-2 infection and account for $<1.5 \%$ of hospitalizations [1]. From the age of 5 years onward, the risk of severe COVID-19 doubles about every 5 years $[2,3]$. A small subset of children develops multisystem inflammatory syndrome in children (MIS-C) around a month after exposure to SARS-CoV-2 [4-6].The prevalence of MIS-C is unknown but estimated to be less than 1 in 50,000 [7]. This severe inflammatory condition is primarily characterized by fever, rash, shock, and cardiac, respiratory, and/or gastrointestinal involvement. MIS-C affects children with a median age of 8.3 years and usually has favorable outcomes after a median hospital stay of 7 days [4, 8, 9]. Risk factors predisposing children to develop MIS-C include being Black and Hispanic [4, 7]. MIS-C pathogenesis is thought to be primarily immunemediated. Children with MIS-C have evidence of broad immune cell activation (phospho-STAT signaling, upregulation of ICAM1, Fc $\gamma$ RI), elevated circulating cytokines (including IL-6, IL-8, and IL-18, among others), and the presence of autoantibodies both against known antigens and novel candidates [6, 10-12].

Individuals with Down syndrome (DS), or trisomy 21, show signs of immune dysregulation, including a higher incidence of autoimmune and autoinflammatory diseases such as autoimmune thyroid disease, autoimmune skin conditions, celiac disease, and type I diabetes [13, 14]. At the molecular level, they have elevated inflammatory markers and an exacerbated response to type I interferon $[15,16]$. Somewhat paradoxically, they are also more susceptible to viral infections, as documented for respiratory syncytial virus and influenza virus $[17,18]$. While epidemiologic studies of COVID-19 hospitalization in DS suggest increased rate, morbidity, and mortality in this population [19], MIS-C has not yet been reported in individuals with DS. Herein, we present cases of two unrelated infant girls with DS who developed a clinical course characterized by symptomatic COVID-19 followed by atypical MIS-C illness requiring hospitalization of over 4 months.

\section{Patient 1}

A 6-month-old North African girl with DS and congenital heart disease had mild COVID-19 manifestations including fever, fatigue, and cough after her father had contracted COVID-19. She received a positive RT-PCR test in March 2020. Ten days later, she was admitted to the pediatric intensive care unit (PICU) due to heart dysfunction and distributive shock. She tested negative by RT-PCR at this time. During her stay, the patient had a generalized maculopapular erythematous rash with peripheral desquamation, edematous hands, vomiting, persistent fever, and eventually coronary dilatation. She received lopinavir/ritonavir, hydroxychloroquine, high-dose intravenous immunoglobulin (IVIG), corticosteroids, and azithromycin. She was diagnosed with MIS-C with myocarditis signs and coronary dilatation. Despite requiring a 4-month hospitalization in the PICU complicated by multiple catheter-related bloodstream infections, the final outcome was favorable. Blood samples were obtained in July 2020, while the patient was afebrile but still in the PICU.

\section{Patient 2}

An 8-month-old Hispanic girl with DS, atrioventricular canal defect status post repair, pulmonary hypertension with chronic oxygen dependence, and nasogastric tube feed dependence was admitted to the PICU for respiratory failure secondary to RTPCR positive COVID-19 pneumonia in October 2020. She was treated with remdesivir, COVID-19 convalescent plasma, and corticosteroids and had waxing and waning respiratory distress and afebrile episodes that lasted up to 5 days. Two weeks after initial onset of COVID-19 symptoms, her fever returned accompanied by elevated inflammatory markers, anemia, thrombocytopenia, elevated ferritin, hypertriglyceridemia, low fibrinogen, and elevated liver function tests. Her respiratory condition worsened, and she developed a diffuse blanching erythematous rash. A second RT-PCR test in December 2020 was negative. At this time, she was IgG positive. Hemophagocytic lymphohistiocytosis (HLH) was initially considered as the patient had elevated IL-2 receptor and low natural killer cell function, but she had normal perforin/granzyme B and her bone marrow aspiration did not show hemophagocytosis. She was treated with IVIG, higher dose corticosteroids, and Anakinra with no improvement in symptoms. She became critical with a prolonged hospitalization complicated by multiple infections, multi-organ failure, and distributive shock requiring vasopressor support. Repeat bone marrow aspiration revealed hemophagocytosis, so she was started on etoposide. With no improvement on etoposide, emapalumab and ruxolitinib were trialed. The patient died in April 2021 after succumbing to gram negative sepsis. Blood samples were obtained in January 2021 while the patient was in the PICU in critical condition on corticosteroids.

\section{Results}

Whole blood immune phenotyping by mass cytometry revealed neutrophilia and a profound decrease in total B cells in both patients compared to uninfected age-matched children with DS $(p=0.008)$ (who already had a decrease in total B cells compared to uninfected without DS, $p=0.002$ ) (Fig. 1A,B). Within total $\mathrm{B}$ cells, memory and plasma $\mathrm{B}$ cell subsets were decreased 
in both patients compared to controls, while naïve B cells were relatively increased (Fig. 1C). These findings in patients with DS months after onset of disease differed from patients without DS with acute MIS-C which were reported previously, who had normal $B$ cell counts $[6,11]$. We also found a depletion of activated $\left(\mathrm{CD} 38^{+} \mathrm{HLADR}^{+}\right) \mathrm{CD} 4 \mathrm{~T}$ cells in our patients, likely suggesting extravasation to the affected tissues (Fig. 1D). Interestingly, we found that activated $\left(\mathrm{CD} 38^{+} \mathrm{HLADR}^{+}\right) \mathrm{CD} 8 \mathrm{~T}$ cells were also depleted in P1, while they were elevated in P2 (Fig. 1E). Previous studies of non-DS patients with MIS-C report either unchanged or elevated activated T cells $[6,11]$. Importantly, we confirm that the immune state of uninfected children with DS is significantly altered [20], including already decreased B cells, increased NK cells, and slightly elevated myeloid dendritic cells (Fig. 1B). It remains unknown if these altered subsets contributed to susceptibility to severe disease after SARS-CoV-2 infection.

We then surveyed markers of inflammation and detected increased STAT3 phosphorylation in most immune subsets in $\mathrm{P} 1$ and in $\mathrm{CD} 4^{+}$TEMRA, NK cells, CD16 ${ }^{+}$monocytes, and eosinophils in $\mathrm{P} 2$, compared with uninfected controls with or without DS (Fig. 1F). This was similar to augmented phospho-STAT3 in patients with acute MIS-C without DS [6]. Furthermore, intracellular interleukin-8 (IL-8) and interleukin-1 $\beta$ (IL-1 $\beta$ ), two pro-inflammatory mediators that enhance neutrophil recruitment and cytokine release, respectively, were elevated in P1's neutrophils and CD16 ${ }^{+}$ and $\mathrm{CD} 14^{+}$monocytes even after clinical signs of MIS-C had resolved (Fig. 1G,H) but not in P2. Discrepancies in inflammatory markers between $\mathrm{P} 1$ and $\mathrm{P} 2$ may be due to the fact that P2 was on high-dose steroids at the time of sampling. Additionally, clinical laboratory inflammatory markers including CRP, ferritin, procalcitonin, and LDH were highly elevated in both patients (Table 1). Together, these data indicate a broad, nonspecific, immune activation in these two patients with DS after SARS-CoV-2 infection compared with age-matched uninfected individuals with or without DS.

Interestingly, other markers of immune activation such as ICAM1/CD54 and FcyRI/CD64 were found to be elevated in myeloid cells not only in P1 and P2, but also in uninfected children with DS (Fig. 1I-L). This elevation was comparable to that in non-DS children with MIS-C. These markers were not detected in children without DS or MIS-C. ICAM1 mediates immune cell extravasation into tissues, and FcyRI can trigger potent inflammation upon antibody binding. Furthermore, the two broad-acting pro-inflammatory cytokines interleukin-6 (IL-6) and IL-8 were elevated in the serum of P1 and $\mathrm{P} 2$, as well as in uninfected children with DS (Fig. 1M,N). This confirms cytokine dysregulation previously reported at baseline in children with DS [15, 23]. Collectively, these findings suggest that children with DS are in a state of immune activation, even in the absence of a pathogen.

\section{Conclusion}

We show that severe, atypical MIS-C can occur in children with DS. These two patients fulfilled the clinical criteria for MIS-C diagnosis [9], and their immunological profile resembled that of previously described children with MIS-C without DS, including neutrophilia, lymphopenia, elevated phosphoSTAT3, ICAM-1, Fc $\gamma$ RI, and circulating cytokines. By contrast, their significant B cell depletion, as well as their young age, prolonged hospital stay, and refractoriness to treatment in $\mathrm{P} 2$, set them apart from children with MIS-C without DS. Of note, the patients' samples were collected months after initial onset while children with MIS-C were sampled within weeks of onset. This study and a recent case report also describing severe disease after SARS-CoV-2 infection in two other children [24] emphasize the notion that children with DS are at unique risk of atypical disease presentation. Further research is needed to elucidate mechanisms underlying disease progression and tailored treatment protocols in this patient population. This report suggests that vaccination should be considered in children with DS for prevention of SARS-CoV-2 infection.

Importantly, uninfected children with DS already had elevated markers of immune activation at baseline, including ICAM1, Fc $\gamma$ RI, and serums IL-6 and IL-8. Together with previously reported defects in immune activation in DS [16, 20,22], our report highlights that DS should be considered an inborn error of immunity, in the broad sense of the term, not restricted to monogenic disorders. Although no clear mechanistic or epidemiologic links between DS and the predisposition to MIS-C have been reported yet, we describe a pre-existing inflammatory state in pediatric DS patients that could have contributed to the severe course of MIS-C in our two patients. As these first reports of severe autoinflammation brought on by SARS-CoV-2 in DS may indicate a larger looming problem with novel syndromic features, we suggest particular care should be taken to children with DS during the COVID-19 pandemic.

\section{Methods}

\section{Sample Collection}

The study was approved under the protocols Mount Sinai Health System (MSHS) IRB-18-00,638 and Vall d'Hebron Research Institute, Barcelona, Spain, PR(AG)198/2014. All patient data were de-identified. Written informed consent for all individuals in this study was provided in compliance with an institutional review board protocol. All uninfected patient samples were drawn in the context of an outpatient routine visit, and patients exhibited no signs of infection (fever, runny nose, cough, sore throat). From each patient, 


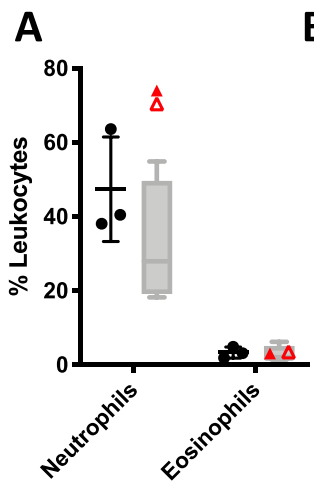

D

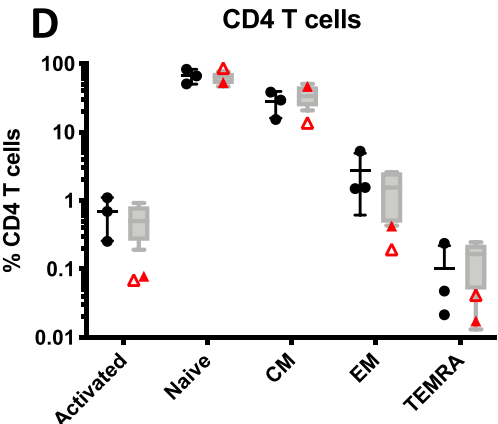

$$
\mathbf{F}
$$

F

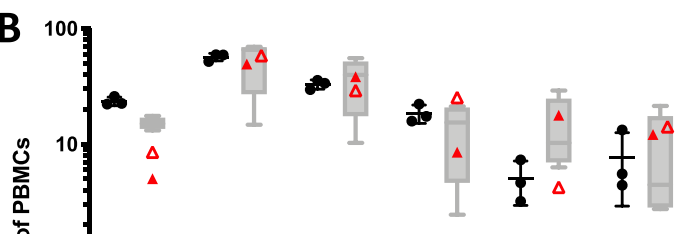

.

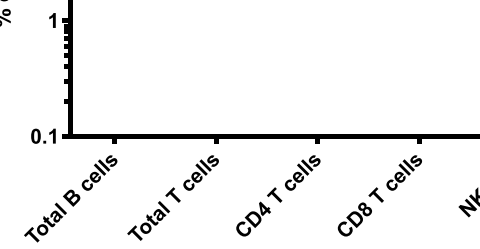

E

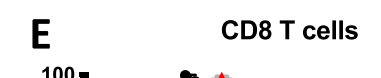

- $H C(n=3)$
DS uninfected

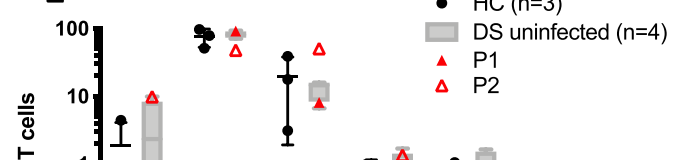

DS uninfected 1 DS uninfected 2 $\mathrm{HC}$
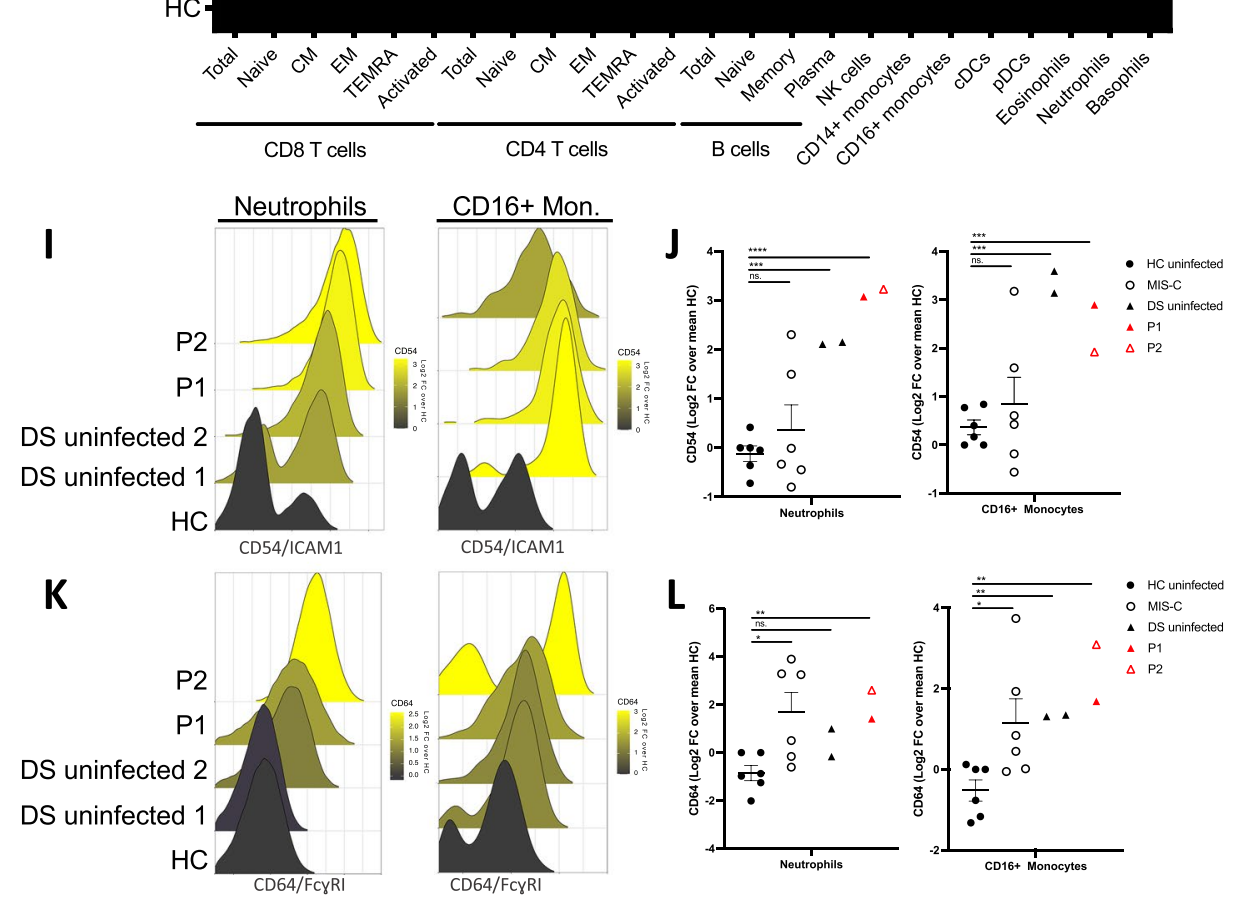

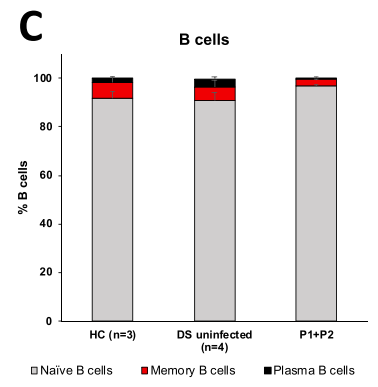

G

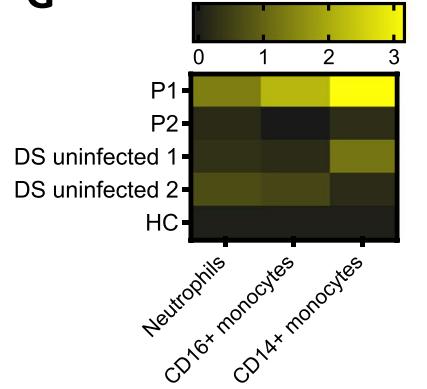

H

IL-8 (Log2 FC over HC)

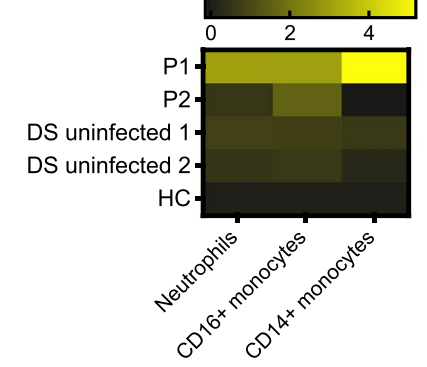

M

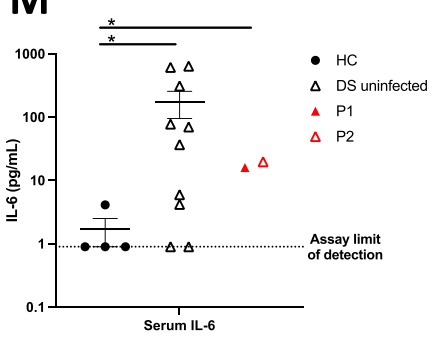

$\mathbf{N}$

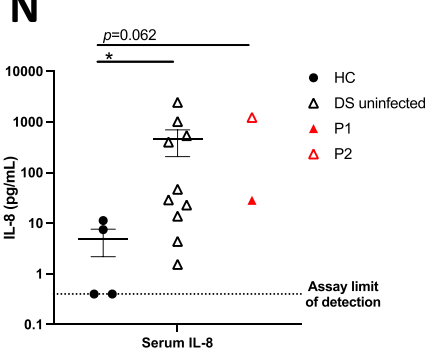


4Fig. 1 Immune phenotyping by mass cytometry of peripheral blood from P1 and P2 and age-matched controls with or without DS. A Granulocyte cell type frequencies as percent of CD45+cells (leukocytes). B Major cell type frequencies as percent of CD66b- cells (nongranulocytes). C B cell, D CD4, and E CD8 T cell frequencies. F STAT3 phosphorylation across immune cell subtypes. G,H Intracellular IL-8, IL-1 $\beta$, cytokine staining and I-L extracellular CD54, and CD64 expression in neutrophils and monocyte subsets, color-coded by the mean $\log 2$ transformed signal intensity. Differences between groups were assessed in unpaired t tests. ${ }^{*} \mathrm{p} \leq 0.05 ; * * \mathrm{p} \leq 0.005$; $* * * \mathrm{p} \leq 0.0005 ; * * * * \mathrm{p} \leq 0.00005$. M,N IL-6 and IL-8 levels in patient serum. Differences between groups were assessed in unpaired t tests of $\log 10$ values

blood was drawn into a cell preparation tube with sodium heparin (BD Vacutainer). Plasma was isolated from using Ficoll separation and stored at $-80{ }^{\circ} \mathrm{C}$ until use. Whole blood was fixed using Proteomic Stabilizer PROT1 (SmartTube) and frozen at $-80^{\circ} \mathrm{C}$. Controls range from 7 months to 6 years old in immune phenotyping studies (Fig. 1A-E), from 19 months to 2 years in inflammatory marker expression analyses (Fig. 1F-I,K), and from 7 months to 19 years old in Luminex analyses (Fig. 1M,N).

\section{Mass Cytometry}

Frozen stabilized blood samples were thawed according to the manufacturer's recommended protocol and then washed with barcode permeabilization buffer (Fluidigm). Samples were uniquely barcoded with Cell-ID 20-Plex Pd Barcoding Kit (Fluidigm) and washed and pooled together. An Fc-block and a heparin-block were then performed to prevent non-specific binding. Cells were then incubated with an antibody cocktail for surface markers to identify major immune populations. All antibodies were purchased with pre-conjugated or conjugated in-house with X8 MaxPar conjugation kits (Fluidigm). After surface staining, the samples were methanol-permeabilized, washed, heparin-blocked, and stained with a cocktail of antibodies against intracellular targets, including markers of phosphorylation and signaling. After washing, cells were then incubated in freshly diluted $2.4 \%$ formaldehyde containing $125 \mathrm{nM}$ Ir Intercalator (Fluidigm), 0.02\% saponin and $30 \mathrm{nM}$ OsO4 (ACROS Organics) for $30 \mathrm{~min}$ at room temperature. Samples were then washed and acquired immediately.

For acquisition, samples were washed with $\mathrm{PBS}+0.2 \%$ BSA, PBS, and then CAS buffer (Fluidigm). The final solution in CAS buffer consisted of 1 million cells per $\mathrm{mL}$ and a 1/20 dilution of EQ beads (Fluidigm). Following routine instrument optimization, samples were acquired at a rate of $<300$ events per second on a Helios mass cytometer (Fluidigm) with a modified wide-bore injector (Fluidigm).

FCS files of acquired events were normalized and concatenated with Fluidigm acquisition software and deconvoluted with a MATLAB-based debarcoding application, and resulting files were analyzed using Cytobank. Cell events were identified as Ir191/193-positive and Ce140-negative events. Doublets were excluded on the basis of Mahalanobis distance and barcode separation and with the Gaussian parameters calculated by Helios CyTOF software. Downstream data analysis was performed on Cytobank, by biaxial gating of immune populations according

Table 1 Clinical characteristics of P1, P2, and children with MIS-C or DS

\begin{tabular}{|c|c|c|c|c|}
\hline & Children with MIS-C [4-9] & Children with DS & $\mathrm{P} 1$ & $\mathrm{P} 2$ \\
\hline Age & Median 8.3 years [4] & $\mathrm{N} / \mathrm{A}$ & 6 months & 8 months \\
\hline Evidence of SARS-CoV-2 & $\begin{array}{l}\text { Positive RT-PCR and/or } \\
\text { serology }\end{array}$ & Absent & $\begin{array}{l}\text { Positive RT-PCR } \\
\text { Negative serology }\end{array}$ & $\begin{array}{l}\text { Positive RT-PCR } \\
\text { Positive serology }\end{array}$ \\
\hline \multicolumn{5}{|l|}{ Clinical presentation } \\
\hline COVID-19 symptoms & Absent & Absent & Fever, fatigue, cough & Fever, COVID-19 pneumonia \\
\hline MIS-C symptoms & $\begin{array}{l}\text { Fever, rash, shock, puffy } \\
\text { extremities, conjunctivitis, } \\
\text { and/or mucocutaneous, } \\
\text { gastrointestinal, cardiac, } \\
\text { respiratory abnormalities }\end{array}$ & Absent & $\begin{array}{l}\text { Fever, rash, shock, periph- } \\
\text { eral desquamation, puffy } \\
\text { hands, vomiting, cardio- } \\
\text { vascular abnormalities }\end{array}$ & $\begin{array}{l}\text { Fever, rash, shock, respira- } \\
\text { tory failure, elevated liver } \\
\text { enzymes }\end{array}$ \\
\hline Length of hospitalization & $\begin{array}{l}\text { Median } 7 \text { days (interquartile } \\
\text { range, } 4 \text { to } 10 \text { days) [4] }\end{array}$ & $\mathrm{N} / \mathrm{A}$ & 4 months & 6 months \\
\hline Laboratory findings & $\begin{array}{l}\uparrow \uparrow \text { CRP, ESR, IL-6, procal- } \\
\text { citonin, ferritin, LDH } \\
\uparrow \uparrow \text { Fibrinogen and D-dimer } \\
\text { Hypoalbuminemia } \\
\uparrow \uparrow \text { Troponin } \\
\uparrow \text { AST, ALT } \\
\text { Lymphopenia, neutrophilia }\end{array}$ & $\begin{array}{l}\uparrow \text { CRP, IL-6 [21] } \\
\text { Lymphopenia [22] }\end{array}$ & $\begin{array}{l}\uparrow \uparrow \text { CRP, ferritin, IL-6, } \\
\text { procalcitonin, LDH } \\
\uparrow \uparrow \text { Fibrinogen and D-dimer } \\
\text { Hypoalbuminemia } \\
\uparrow \uparrow \text { Troponin } \\
\text { Lymphopenia, neutrophilia }\end{array}$ & $\begin{array}{l}\uparrow \uparrow \text { CRP, ferritin, IL-6, proc- } \\
\quad \text { alcitonin, LDH } \\
\uparrow \uparrow \text { Fibrinogen and D-dimer } \\
\text { Hypoalbuminemia } \\
\text { Normal Troponin } \\
\uparrow \text { AST, ALT } \\
\text { Lymphopenia, neutrophilia }\end{array}$ \\
\hline Immune activation & $\begin{array}{l}\uparrow \text { phospho-STAT3 } \\
\uparrow \text { FcyRI and ICAM1 on } \\
\text { neutrophils, monocytes } \\
\uparrow \text { Serum IL-6, IL-8 }\end{array}$ & $\begin{array}{l}\uparrow \text { FcyRI and ICAM1 on } \\
\text { neutrophils, mono- } \\
\text { cytes } \\
\uparrow \text { Serum IL-6, IL-8 }\end{array}$ & $\begin{array}{l}\uparrow \text { phospho-STAT3 } \\
\uparrow \text { FcyRI and ICAM1 on } \\
\text { neutrophils, monocytes } \\
\uparrow \text { Serum IL-6, IL-8 }\end{array}$ & $\begin{array}{l}\uparrow \text { FcyRI and ICAM1 on } \\
\text { neutrophils, monocytes } \\
\uparrow \text { IL-2R } \\
\uparrow \text { Serum IL-6, IL-8 }\end{array}$ \\
\hline
\end{tabular}


to the gating scheme outlined in Supplemental Fig. 1. All data points represent a single patient. For samples processed at different time points (batch 1, HC01, DS01, DS02, P1; batch 2, HC01, DS01, P2), a batch correction strategy was applied by normalizing relevant markers (p-STAT3, IL-1b, IL-8, CD54, CD64) to a common control (HC01).

\section{Multiplex Cytokine Analysis}

Plasma collected by Ficoll isolation from heparinized whole blood was clarified by centrifugation. Circulating cytokine levels were determined for P1, P2, pediatric controls without DS $(n=4)$, and pediatric uninfected controls with DS $(\mathrm{n}=10)$. Magnetic Luminex assays with the MILLIPLEX MAP Human Cytokine/Chemokine Magnetic Bead Panel (MilliporeSigma HCYTMAG-60 K-PX30) were run according to the manufacturer's protocol. Samples were quantified on a MAGPIX xMAP Instrument (Luminex).

\section{Statistics}

Unpaired $t$ tests were used to assess the significance of differences in quantitative variables between two groups.

Supplementary Information The online version contains supplementary material available at https://doi.org/10.1007/s10875-021-01078-4.

Acknowledgements We thank Adeeb Rahman, Daniel Geanon, and Geoffrey Kelly from the Human Immune Monitoring Center at the Icahn School of Medicine for their technical assistance.

Funding This research was supported by the National Institute of Allergy and Infectious Diseases Grants R01AI150300 and R01AI150300-01S1. LM was supported by T32 training grant T32HD075735.

\section{Declarations}

Ethics approval The study was approved under the protocols Mount Sinai Health System (MSHS) IRB-18-00638 and Vall d'Hebron Research Institute, Barcelona, Spain, PR(AG)198/2014. All patient data were de-identified.

Conflict of Interest The authors declare no competing interests.

\section{References}

1. American Academy of Pediatrics and Children's Hospital Association, Children and COVID-19: State-Level Data Report. 2021. https://servi ces.aap.org/en/pages/2019-novel-coronavirus-covid-19-infections/ children-and-covid-19-state-level-data-report/. Accessed April 2021.

2. O'Driscoll M, et al. Age-specific mortality and immunity patterns of SARS-CoV-2. Nature. 2021;590:140-5.

3. Levin AT, et al. Assessing the age specificity of infection fatality rates for COVID-19: systematic review, meta-analysis, and public policy implications. Eur J Epidemiol. 2020;35:1123-38.
4. Feldstein LR, et al. Multisystem inflammatory syndrome in U.S. children and adolescents. N Engl J Med. 2020;383:334-46.

5. Ahmed M, et al. Multisystem inflammatory syndrome in children: a systematic review. EClinicalMedicine. 2020;26:100527.

6. Gruber $\mathrm{CN}$, et al. Mapping systemic inflammation and antibody responses in multisystem inflammatory syndrome in children (MIS-C). Cell. 2020;183:982-995.e14.

7. Sancho-Shimizu V, Brodin P, Cobat A, Biggs CM, Toubiana J, Lucas CL, et al. SARS-CoV-2-related MIS-C: A key to the viral and genetic causes of Kawasaki disease? J Exp Med. 2021;218(6):e20210446. https://doi.org/10.1084/jem.20210446.

8. Multisystem inflammatory syndrome in children and adolescents temporally related to COVID-19. https://www.who.int/newsroom/commentaries/detail/multisystem-inflammatory-syndromein-children-and-adolescents-with-covid-19. Accessed April 2021.

9. HAN Archive - 00432 I Health Alert Network (HAN). https://emergency. cdc.gov/han/2020/han00432.asp. Accessed April 2021.

10. Consiglio CR, et al. The immunology of multisystem inflammatory syndrome in children with COVID-19. Cell. 2020;183:968-981.e7.

11. Vella LA, et al. Deep immune profiling of MIS-C demonstrates marked but transient immune activation compared to adult and pediatric COVID-19. Sci Immunol. 2021;6:eabf7570.

12. Ramaswamy A, et al. Post-infectious inflammatory disease in MIS-C features elevated cytotoxicity signatures and autoreactivity that correlates with severity. medRxiv 2020; 2020.12.01.20241364. https://doi.org/10.1101/2020.12.01.20241364.

13. Bull MJ. Down syndrome. N Engl J Med. 2020;382:2344-52.

14. Giménez-Barcons M, et al. Autoimmune predisposition in Down syndrome may result from a partial central tolerance failure due to insufficient intrathymic expression of AIRE and peripheral antigens. J Immunol. 2014;193:3872-9.

15. Sullivan KD, et al. Trisomy 21 causes changes in the circulating proteome indicative of chronic autoinflammation. Sci Rep. 2017. https://doi.org/10.1038/s41598-017-13858-3.

16. Kong X-F, et al. Three copies of four interferon receptor genes underlie a mild type I interferonopathy in Down syndrome. J Clin Immunol. 2020. https://doi.org/10.1007/s10875-020-00803-9.

17. Löwensteyn YN, et al. Respiratory syncytial virus-related death in children with down syndrome: the RSV GOLD Study. Pediatr Infect Dis J. 2020;39:665-70.

18. Pérez-Padilla R, et al. Pandemic (H1N1) 2009 Virus and Down syndrome patients. Emerg Infect Dis. 2010;16:1312-4.

19. Malle L, et al. Individuals with Down syndrome hospitalized with COVID-19 have more severe disease. Genet Med. 2020. https:// doi.org/10.1038/s41436-020.

20. Huggard D, Doherty DG, Molloy EJ. Immune dysregulation in children with Down syndrome. Front Pediatr. 2020;8:1-10.

21. Licastro F, Chiappelli M, Ruscica M, Carnelli V, Corsi MM. Altered cytokine and acute phase response protein levels in the blood of children with Downs syndrome: relationship with dementia of Alzheimer's type. Int J Immunopathol Pharmacol. 2005;18:165-72.

22. Verstegen RHJ, Kusters MAA. Inborn errors of adaptive immunity in Down syndrome. J Clin Immunol. 2020;40:791-806.

23. Zhang Y, et al. Aberrations in circulating inflammatory cytokine levels in patients with Down syndrome: a meta-analysis. Oncotarget. 2017;8:84489-96.

24. Kantar A, et al. COVID-19 and children with Down syndrome: is there any real reason to worry? Two case reports with severe course. BMC Pediatr. 2020;20:1-5.

Publisher's Note Springer Nature remains neutral with regard to jurisdictional claims in published maps and institutional affiliations. 